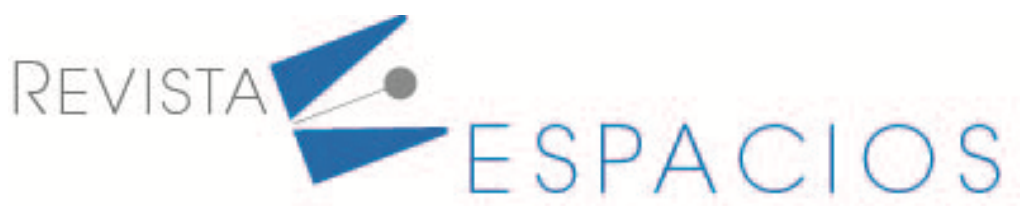

\title{
El proceso de formación investigativa del ingeniero industrial basado en competencias
}

\section{The industrial engineer research formation process based on competences}

\author{
SÁNCHEZ, Pedro R. ${ }^{1}$ \\ CAICEDO, Eduardo A. ${ }^{2}$ \\ PATIÑO, María C. ${ }^{3}$
}

\begin{abstract}
Resumen
El propósito esencial de este artículo es brindar una concepción teórica del proceso de formación investigativa del ingeniero industrial basada en competencias, en el mismo destacan las razones esenciales conducentes a la adopción de este enfoque como alternativa para lograr favorecer el desempeño investigativo de estos profesionales, entre las que sobresale la relativa a los principios que incrementan el potencial de adaptabilidad al cambio del sujeto formado bajo esta perspectiva, además se abordan las características generales de la formación investigativa vista como proceso y los rasgos que permiten catalogarla como resultado.
\end{abstract}

Palabras clave: competencia investigativa; formación investigativa; desempeño investigativo

\begin{abstract}
The essential purpose of this article is present a theoretical conception of the industrial engeneer' $\mathrm{s}$ research formation process based on competences. In this paper the main arguments supporting this approach as an alternative to favor these professional' $s$ research performance are exposed emphasizing the formulation of principles that increase the subject's potentiality of change adaptability. Further more the research formation general characteristics are dealt with both as a process and the features that allow to consider then as results.
\end{abstract}

Key words: research competence; research formation; research performance

\section{Introducción}

El pasado siglo mostró significativos avances científicos e innovaciones tecnológicas que aceleraron y revolucionaron el desarrollo de la humanidad y el actual se muestra, en sus inicios, no menos promisorio en cuanto a la continuidad del progreso en todas las ramas del saber.

La creación acelerada de conocimientos, pero también su rápida obsolescencia, plantean el reto de una necesaria y permanente renovación de los mismos en un entorno social, laboral y económico con una dinámica sorprendente y en constante evolución como resultado de los propios avances científicos y tecnológicos.

\footnotetext{
${ }^{1}$ Universidad de Holguín. Email: pramonst230761@gmail.com

2 Universidad Laica Eloy Alfaro de Manabí. Email: eduardo.caicedo@uleam.edu.ec

${ }^{3}$ Universidad Laica Eloy Alfaro de Manabí. Email: maria.patino@uleam.edu.ec
} 
Al hacer referencia a la obra La sociedad Global de Chomsky y Dieterich (1997), en lo concerniente al papel de los conocimientos y la educación en el presente siglo, J. Núñez, J. (1999) afirma: "En la economía global del siglo XXI la calificación científica y profesional de la fuerza de trabajo debe constituir el arma competitiva fundamental, que desplaza las ventajas comparativas tradicionales como riqueza natural u otras."

Los cambios resultantes de los avances científicos hacen necesaria la formación de profesionales con una alta calificación, lo cual ha impactado de manera significativa en el vínculo universidad empresa. La necesidad de relacionar la formación con los requerimientos de las organizaciones productivas no es nueva. Samaniego, N. (1996) hace referencia a que “(...) cada una de estas dos instituciones, escuela y empresa, han seguido propósitos y tiempos distintos y que sus trayectorias han sido a menudo divergentes (...)"

El desempeño es en el mercado de trabajo nacional o internacional el medidor de la calidad, teniendo en cuenta que a los profesionales se les exige adaptabilidad a diversos contextos y culturas, lo que hace necesario un nuevo equilibrio entre la oferta de formación por parte de las instituciones encargadas de ello y la demanda del mercado de trabajo. En este escenario organizacional, si las organizaciones demandan competencias profesionales, las instituciones encargadas de la formación deben favorecer el desarrollo de estas y entregar un egresado que pueda asumir la práctica profesional como una actividad innovadora.

Dadas las características que tiene la llamada "Sociedad del Conocimiento", en la cual este escenario organizacional está inmerso y en la que a decir de Fuentes González, H. (2004) y con lo que se coincide, “(...) crea el valor por la aplicación del conocimiento al trabajo, expresado en términos de productividad e innovación (...)”, todo profesional debe tener una sólida formación investigativa aún cuando no se desempeñe como investigador. "La dinámica y requerimientos actuales de la investigación científica hacen necesario orientarlo hacia la formación de competencias investigativas, lo cual permite vincular la educación con el desempeño real que tendrá el egresado en la vida académica y profesional." Cruz Baranda, S. y Fuentes González, H. (2003).

Estas ideas son reafirmadas para el profesional de la ingeniería por algunas experiencias realizadas en el campo de la Ciencia-Tecnología-Sociedad, donde Castro Sánchez, F. (1999) plantea que

(...) el trabajo del ingeniero no despliega todas sus potencialidades para la sociedad si sus funciones las reduce a las de un técnico que sólo interviene en la aplicación de resultados. Él ha de trabajar con las llamadas nuevas tecnologías que no dejan lugar al éxito sin un conocimiento básico profundo. Entonces ha de proponerse alcanzar en toda su medida la condición investigativa y de autosuperación propias del hombre de ciencia (...)

Sustentar el binomio hombre-empresa requiere que los procesos educativos y formativos se vinculen con los procesos de producción de bienes y servicios, haciendo énfasis marcado en la resolución de problemas profesionales, que hagan más sólida y competitiva la posición de la organización. El reto para las organizaciones es según Moreno González, y Rodríguez González (2002),“(...) generar valor a través de la creación de conocimiento." Las organizaciones demandan profesionales que sean más que poseedores de conocimientos enciclopédicos, competentes.

Autores como Addine (1997), Martínez Llantada (2000), Salazar Fernández (2001), Barrera Kalhil (2003), Machado Ramírez, Montes de Oca y Menas Campos (2008), han contribuido al perfeccionamiento del proceso de formación investigativa desde la perspectiva de la formación de habilidades investigativas, lo que en los momentos actuales resulta insuficiente si se quiere corresponder a las exigencias del contexto socioprofesional.

Otro grupo de autores abordan la situación planteada con propuestas y resultados sustentados en la formación de competencias investigativas, entre ellos Gallardo Milanés (2002), quien aporta un modelo para la formación 
por competencias de investigadores profesionales y la considera una forma de capacitación profesional, lo que en el contexto de su investigación es correcto; sin embargo, enuncia las competencias como capacidad, lo que le confiere un carácter restringido a su propuesta.

Cruz Baranda y Fuentes González (2004), proponen un sistema de competencias investigativas propio de la formación de doctores; en este caso es posible extrapolar ideas y relaciones aplicables al proceso de formación inicial de profesionales. En estudios posteriores y coincidiendo con el autor de esta investigación, Fuentes (2008) reconoce la competencia como una cualidad humana. También Ruiz (2001), Castellanos Simona y col. (2003), Llivina, y col. (2005), hacen significativos aportes a la conceptualización de la competencia investigativa aunque centrada específicamente en los profesionales de la educación.

Sin embargo, a pesar del gran número de autores involucrados en el estudio de esta temática y de los resultados alcanzados, no se han encontrado aportes relacionados con la formación investigativa basada en competencias para el ingeniero industrial. Además, emerge una precisión del estudio y análisis de sus consideraciones y es que se requiere un proceso de formación investigativa que tome en cuenta las potencialidades y exigencias del entorno socioprofesional en que se desarrolla para que los estudiantes puedan alcanzar la idoneidad en su desempeño investigativo. Se debe destacar que entre las exigencias sobresale la demanda de profesionales "competentes".

\subsection{El contexto formativo y las carencias teóricas fundamentales}

La concepción de la formación investigativa abordada, trata que el estudiante al iniciar su vida laboral sea capaz de resolver los problemas que surjan en su entorno socioprofesional, empleando para ello métodos científicos, "(...) lo laboral en un graduado universitario hay que entenderlo siempre desde una perspectiva científica." Horruitiner Silva, P. (2006)

Estudios realizados por Barrera Kalhil, J. (2003) muestran que:

(...) una de las vías utilizadas para tratar de resolver este problema, es la incorporación al Plan de estudio en las carreras universitarias de la asignatura Metodología de la investigación, la que involucrada de modo atomizado con relación (...) al propio proceso de formación del futuro egresado no responde al reto que impone la sociedad actualmente de un profesional integral, mucho menos es garantía para que se produzca la relación dialéctica profunda entre los componentes organizacionales del currículo, académico, investigativo y laboral.

Aunque esta vía de solución está en vigor en las universidades, el problema persiste, según lo corroboran los resultados obtenidos en una investigación realizada por el Centro de Estudios para el Perfeccionamiento de la Educación Superior (CEPES) y por la Dirección de Formación Profesional del Ministerio de Educación Superior (MES) referente a la calidad del graduado. En este se indagaron aspectos relacionados con el desempeño investigativo de los estudiantes y egresados de la educación superior en general, y evidenció que a pesar del énfasis puesto en lograr el desempeño deseado existen insuficiencias investigativas tales como:

- Insuficiente preparación para la búsqueda y uso de la información actualizada.

- Insuficiente dominio de conocimientos de metodología y práctica de la investigación científica.

A su vez, estudios recientes realizados por Coello González y Hernández León (2008), evaluaron los conocimientos mínimos que debían poseer los estudiantes universitarios de diferentes carreras de las ciencias técnicas en Cuba, sobre conceptos fundamentales para el proceso de investigación y mostraron que el 86,6 \% 
(200) de los 232 conceptos evaluados fueron calificados de mal, en el caso específico de los estudiantes de Ingeniería Industrial.

Las conclusiones de esta investigación aseveran que:

- Los egresados de la educación superior no poseen la información mínima que necesitan como investigadores para trabajar en un medio donde se investiga desde la producción.

- Los programas que se imparten actualmente no permiten formar todas las habilidades que se necesitan para investigar.

Otros documentos cuya revisión aportó evidencias relativas a la insuficiente formación investigativa recibida, son los referentes a la aprobación y ejecución de proyectos en la Delegación Provincial del Ministerio de Ciencia, Tecnología y Medio ambiente (CITMA - Holguín ), los informes correspondientes al Balance de Ciencia e Innovación Tecnológica de la carrera de Ingeniería Industrial, y el Plan de Ciencia e Innovación Tecnológica, los que permitieron constatar la baja participación de ingenieros industriales que ejercen la profesión en proyectos, resultado que está vinculado con los proyectos desarrollados por la Facultad de Ingeniería Industrial, o sea, no se pudo determinar la existencia de proyectos gestionados por profesionales de la Ingeniería Industrial en ejercicio, desde la producción y los servicios. Esto evidenció:

- Insuficiente gestión de proyectos de investigación por parte de los egresados.

A lo antes planteado se añaden los resultados aportados por el análisis de la información obtenida de la aplicación de encuestas a egresados de la carrera de Ingeniería Industrial, a jefes de estos graduados, y en la propia Universidad de Holguín a estudiantes de la carrera, los que conjuntamente con el análisis efectuado del Diagnóstico de Computación de la Universidad en esta especialidad, permitieron delimitar las insuficiencias más notables encontradas en la formación investigativa:

- Dificultades para identificar, diagnosticar y formular problemas profesionales, resaltando los casos en que estos tengan carácter científico.

- Deficiencias en la presentación de resultados asociados a trabajos científicos.

- Limitaciones en la gestión de la información necesaria para la investigación, con el empleo de las tecnologías de la información y la comunicación.

- $\quad$ Limitaciones en la gestión del conocimiento.

- Limitada formación para realizar la gestión de proyectos de investigación.

Estas limitaciones que presentan estudiantes y egresados al asumir tareas del quehacer científico, expresan una insuficiente práctica educativa, que se sintetiza como dificultad en una relación contradictoria visible en el plano externo, la que se plantea como: no correspondencia entre las exigencias del contexto socioprofesional en la solución de problemas profesionales inherentes a los procesos básicos de la Ingeniería Industrial (Gestión de la Calidad, Logística Empresarial, Gestión de Procesos y Gestión de Recursos Humanos) y la formación investigativa recibida por los estudiantes en la carrera.

A partir de la investigación realizada se establece como objetivo de la investigación: Elaborar un modelo, y la estrategia que lo concreta, para favorecer lla formación investigativa basada en competencias del estudiante de la carrera de Ingeniería Industrial. 


\subsection{Sobre el modelo y la estrategia}

Un elemento de partida para la modelación de la formación investigativa basada en competencias en la carrera de Ingeniería Industrial lo constituye la teoría general de los sistemas desarrollada por Ludwig von Bertalanffy en el siglo XX. Consecuente con ella se asume el concepto dado por Fuentes, Matos y Montoya (2007) quienes plantean que:

"Un sistema es un conjunto de objetos (procesos) relacionados entre sí por alguna forma de interacción, que los identifica con determinada independencia y coherencia, donde los objetos o procesos adquieren el significado de elementos componentes y sus relaciones determinan el significado alrededor del cual se integran estos, a la vez que los elementos componentes le aportan sentido al sistema. En la determinación del sistema se revelan las relaciones entre los elementos componentes y el comportamiento del todo".

Otros aspectos esenciales que se valoran son las concepciones sobre modelos sustentados en el enfoque de formación basada en competencias, concebidos en investigaciones internacionales (UNESCO 1972, 1983, 2000); los trabajos de especialistas de la Organización de Estados Iberoamericanos (OEI), CEPAL, el Servicio Nacional de Aprendizaje (SENA, Colombia), el Instituto Argentino de Capacitación Profesional y Tecnológica para el Comercio (INACAP), CONOCER (2002 - 2008), los de Huerta y colaboradores (2000), Mertens (2001) y las diferentes experiencias en la formación de trabajadores por competencias, publicadas por CINTERFOR/OIT (2000-2007).

También se tuvieron en cuenta resultados de investigadores como: Ortiz (2001), Roca (2001), González (2002), Fuentes (2002-2004), Cejas (2004), Forgas (2004), Tejeda (2006), Alonso (2007), los que en su conjunto aportaron referentes teóricos, conceptuales y metodológicos significativos de la formación basada en competencias.

Estos antecedentes conducen a considerar las competencias investigativas como un sistema que sustenta la formación investigativa basada en competencias como el resultado del proceso de formación de la personalidad del profesional dirigido a lograr un desempeño investigativo de umbral de idoneidad en el recién graduado e idóneo una vez superada esta etapa y que debe perdurar mediante la formación continua.

El proceso de formación investigativa basada en competencias se sustenta, además, en el enfoque personológico que tiene por presupuesto la teoría humanista y la concepción histórico cultural del desarrollo humano de Vigotsky, del cual se asumen conceptos tales como la mediación, la zona de desarrollo próximo y el carácter social del desarrollo.

Cuando se habla de formar competencias, de hecho y de acuerdo con el propio concepto se está hablando de configurar una cualidad en los sujetos con un carácter holístico, en el que, habilidades, hábitos, actitudes, aptitudes y valores se sintetizan en una unidad dialéctica, de acuerdo con el principio de unidad de lo cognitivo y afectivo, que le brinda coherencia y armonía a la autorregulación de la conducta del sujeto.

La zona de desarrollo próximo se asume tomando en consideración que las competencias logradas son expresadas en el desempeño y lo connotan, por lo tanto, son identificadores del nivel de formación investigativa real alcanzado por el sujeto cuando este transfiere estas a una situación análoga.

Si se tiene en cuenta que existen y se han determinados varios niveles de formación investigativa y que, además, dada la infinitud del conocimiento científico las competencias investigativas que gradan estos niveles están en constante evolución y desarrollo, en determinado momento constituyen el nivel de desarrollo potencial para la consecución con determinado nivel de ayuda (aportado por algún otro actor del proceso, profesor, profesional vinculado al proceso, otros estudiantes) del nivel real de desarrollo. 
Al concebir el modelo se reconoce la contextualización de las exigencias formativas de la carrera de Ingeniería Industrial en los distintos contextos universitarios, ya sean aulas, laboratorios o los laborales profesionales (organizaciones asociadas a la profesión).

Actualmente las universidades están siendo consideradas estructuras dinamizadoras del proceso innovador, entre otros aspectos por su responsabilidad en la formación de los profesionales que trabajarán en organizaciones que consideran la investigación como una parte importante del proceso productivo, por lo que la actividad investigativa es considerada una actividad profesional y es vista como una actividad productiva que exige de los profesionales competencia investigativa.

La lógica del razonamiento científico seguida al construir el modelo transita por distintos momentos, valorados y modificados sobre la propuesta original realizada por Concepción y Rodríguez (2005).

\subsubsection{El proceso o actividad objeto de transformación}

El análisis epistémico en relación con la formación investigativa del ingeniero industrial, permitió revelar carencias teóricas y metodológicas que apuntan a la necesidad de transformar el proceso de formación inicial en lo investigativo, transitando de la formación de habilidades para la investigación a la formación de competencias investigativas, para garantizar una preparación más integral de los estudiantes.

Tomando en consideración, además, el diagnóstico del estado actual de la formación investigativa de los estudiantes de la carrera de Ingeniería Industrial y la experiencia práctica del investigador, se determina como proceso a transformar el proceso de formación investigativa del ingeniero industrial, a partir de ahora, proceso de formación investigativa del ingeniero industrial basado en competencias, el que se entiende como un subproceso del proceso de formación inicial del profesional dirigido a lograr la interiorización de la cultura científico investigativa propia de la profesión y el desarrollo de las competencias investigativas, integrando los componentes académico, laboral e investigativo, de manera tal que, una vez egresado, el estudiante sea competente en el plano investigativo y manifieste un desempeño investigativo con carácter idóneo, sustentado en la generación de ventajas competitivas, originadas al aplicar los conocimientos a los procesos básicos (Logística Empresarial, Gestión de Recursos Humanos, Gestión de la Calidad, y Gestión de Procesos) de manera innovadora.

En la definición dada se revelan como rasgos esenciales los siguientes:

- Es un proceso de preservación, actualización e interiorización, sustentado en los cambios gnoseológicos, tecnológicos, sociales, etc., que se originan con marcada frecuencia en el ámbito socioprofesional y que involucra a tres actores esenciales: estudiantes, profesores y profesionales en ejercicio.

- Como proceso, transita por diferentes etapas y se va haciendo cualitativamente superior en este tránsito, como resultado de su propia dialéctica.

- En él se da la unidad de lo cognitivo, lo motivacional, lo afectivo y conductual, sintetizado en las competencias investigativas que se configuran.

- Carácter dual subjetivo-objetivo del proceso, subjetivo por cuanto se da en el plano interno del sujeto (intrapsicológico) donde las competencias se configuran como cualidad y existen como potencial para la ejecución exitosa, y objetivo, pues se manifiesta externamente en su desempeño, en su relación con el objeto y con otros sujetos (interpsicológico).

- Se sucede de manera consciente, con la participación activa del sujeto implicado, que asimila información, transforma esta en conocimiento, lo aplica a la práctica, genera nueva información y establece de esta manera ciclos en espiral ascendente que implican desarrollo y significan los niveles en 
su formación, en la misma medida en que se enfrenta a la solución de situaciones y problemas profesionales asociados a los procesos básicos de la ingeniera industrial.

- Está dirigido a la formación investigativa de los estudiantes basada en competencias, lo que implica su participación consciente y voluntaria e involucra tanto el "saber", entendido como conocimiento propio de la profesión, con carácter multidisciplinario y el conocimiento propio del proceso de investigación, lo que sustenta un sólido sistema de acciones generalizables, transferibles, multirreferenciables y emergentes; el "saber hacer", referente a la parte procesal, al desarrollo de la actividad profesional como una actividad investigativa, innovadora y desarrolladora; el "saber estar", como expresión del componente regulador de la conducta asociada a las competencias investigativas, el cual integra los aspectos psicológicos que estimulan, sostienen y orientan el desempeño investigativo del profesional, y el "saber ser", caracterizado por los valores relacionados con la actividad investigativa.

- Propicia un desempeño investigativo además de idóneo, sostenible, pues el hecho de formar competencias, las que se caracterizan por ser transferibles, multirreferenciables, permite estar a tono con los constantes cambios que se suceden en los campos del conocimiento y la tecnología y, por ende, mantener un desempeño idóneo en lo investigativo en el decursar del tiempo.

- Requiere una didáctica participativa y flexible que se contextualiza según las características de los sujetos que participan en la formación y los diversos contextos socioprofesionales asociados a la profesión.

Del análisis hecho a una revisión de los conceptos tratados en el Seminario Internacional sobre Formación de Doctores e Investigadores en Iberoamérica, desarrollado en Salamanca, España, del 28 al 30 de enero de 2002 se destacan algunos aspectos didácticos que se deben tener en cuenta en el proceso de formación investigativa basado en competencias:

- Es muy importante el trabajo en grupos científicos estudiantiles, ya que a través de la influencia del grupo de investigación los estudiantes aceleran su formación investigativa.

- Se debe asociar el proceso formativo al desarrollo de proyectos de investigación en los cuales los estudiantes se involucran directamente y en torno a los cuales se generan los problemas de investigación que las tesis de grado o los trabajos de diploma solucionan.

- Desde una perspectiva académica se debe educar al estudiante en las tareas de creación científica.

- Se establecen las redes de conocimientos a través de la cultura de la comunicación, apoyo y confrontación entre los estudiantes y profesores.

- Se deben estimular enfoques interdisciplinarios, multidisciplinarios y transdisciplinarios.

- No se deben sustentar los perfiles de los egresados en listas de conocimientos puntuales, que resultarían obsoletos a corto plazo. El estudiante debe "aprender a aprender".

- Vincular la educación con el desempeño real que tendrá el egresado en la vida académica y profesional.

- Las competencias requeridas para investigar y realizar un ejercicio profesional de alto nivel son abiertas, difíciles de acotar y en las cuales se puede mejorar incesantemente.

- Las competencias no se pueden anclar a contenidos específicos, porque el conocimiento y la tecnología cambian constantemente.

- Es un proceso sistémico, complejo e interdisciplinario. 
Además de estos aspectos didácticos y de los rasgos esenciales de la formación investigativa basada en competencias vista como proceso, se enuncian como rasgos que la distinguen como resultado los siguientes:

Rasgo indagativo: posibilita la apropiación de la cultura científico - investigativa, necesaria para resolver los problemas profesionales siguiendo la lógica de la investigación científica, además de fundamentar los mismos, caracterizar el objeto que se investiga, procesar y analizar críticamente toda la información existente, relacionarla convenientemente con el objetivo de la investigación que se desarrolla, nutrirse de las experiencias anteriores y establecer los nexos entre el problema profesional y sus antecedentes.

Rasgo argumentativo: hace posible expresar de forma oral y escrita argumentos con base científica y el uso de conceptos que permitan fundamentar juicios y valoraciones que demuestren con seguridad el dominio que se tiene acerca de los presupuestos y de la temática de estudio y la capacidad para la síntesis y la concreción, además de la claridad y coherencia con que se brindan los fundamentos científicos en los que se sustentan las consideraciones a las que se arriban como consecuencia del proceso de solución de problemas profesionales.

Rasgo innovador: posibilita el descubrimiento de los aspectos novedosos en la solución de un problema profesional, de una investigación, relacionar variables significativas del objeto y representar, a través de la abstracción, la forma en que se puede influir en el objeto de investigación con vistas a transformarlo o en la solución de un problema para transitar del estado actual al deseado.

Rasgo gerencial: hace posible la gestión de proyectos, la que se asume con dominio de los factores que permiten a un proyecto de investigación o de ingeniería apuntar a la garantía de su impacto, a la validez de sus hipótesis, a la justificación y pertinencia del problema que se investiga, en fin, a todos aquellos elementos que validan el proyecto en sí.

Rasgo tecnológico: posibilita el acceso y uso consecuente de los medios tecnológicos, emplear la tecnología existente a fin de hacer óptimos los resultados de investigación, o de solución de problemas profesionales, tanto por sus niveles de contrastación y comprobación, como por la rapidez en el procesamiento de los datos e información en general, para lo cual debe valorarse la idoneidad de los medios técnicos disponibles y la manera de incorporarlos eficientemente al proceso investigativo o de solución.

Rasgo axiológico: expresión de los valores relacionados con la realización de la actividad investigativa: exigencia en la aplicación del método científico, disposición para el trabajo en grupos, honestidad científica, responsabilidad científica, compromiso social y político- ideológico.

Rasgo conductual: hace posible la integración de aquellos procesos psicológicos que estimulan, sostienen y orientan el desempeño investigativo del estudiante y del profesional, entre los cuales B. Castellanos (2003) señala las motivaciones predominantes intrínsecas hacia la actividad investigativa en función del mejoramiento de la calidad, la autovaloración del sujeto como competente en la actividad que realiza, expectativas positivas acerca de la idoneidad de su desempeño investigativo.

A excepción de los rasgos axiológicos y conductual, los restantes han sido redefinidos (ahora como rasgos), resumidos y sintetizados a partir de la visión de otros autores que los definen como competencias investigativas.

Una vez determinado el proceso que se transformará y tomando en consideración el análisis epistemológico realizado, los resultados del procesamiento de la información sobre el proceso y la manifestación externa de la contradicción, se procede a determinar los elementos entre los que se expresa la manifestación interna de la contradicción (par contradictorio), llegando así al segundo momento, por el que transita la elaboración de este constructo teórico, la determinación de la contradicción fundamental. 


\subsubsection{La contradicción fundamental}

El contexto socioprofesional está matizado por la ocurrencia de cambios de diversos tipos, considérense: sociales, gnoseológicos, tecnológicos, metodológicos y praxiológicos, los que se manifiestan con mayor fuerza en los sectores de la producción y/o los servicios.

Estos cambios en muchas ocasiones no llegan a conocerse o transferirse a las instituciones encargadas de la formación de los profesionales, no se integran a los currículos de las carreras ni los modifican, o llegan a estos con retardo en el tiempo, en un mundo donde la información y los conocimientos se vuelven obsoletos con rapidez. Esto constituye una debilidad del proceso de formación y en muchas ocasiones provoca que estas instituciones estén desactualizadas $y$, sin embargo, supongan estar ofreciendo una formación acorde con los requerimientos del mundo laboral o al menos con una tendencia positiva a satisfacerlos.

El análisis realizado a la relación contradictoria entre las exigencias del contexto socioprofesional y la formación investigativa, conjuntamente con los restantes elementos citados anteriormente, condujo a la necesidad de modelar la formación de las competencias investigativas del ingeniero industrial desde la relación contradictoria en el plano interno dada entre la interiorización de la cultura científico investigativa y el desempeño investigativo.

La cultura científico investigativa es la base del desempeño investigativo, el cual se corresponde con esta y está directamente relacionado con la interiorización de los saberes asociados a la profesión que ha hecho el sujeto. Al mismo tiempo el desempeño investigativo es la expresión en la praxis de la exteriorización de la cultura científico investigativa, es lo objetivo, el lado práctico de esta.

Este desempeño investigativo cuando es idóneo genera nuevos saberes, que no se incorporan de manera inmediata a la cultura (actualmente como resultado de la internación de las investigaciones en los procesos de producción existe una tendencia a utilizar el conocimiento en los mismos lugares donde se produce), en muchas ocasiones esta demora no depende de su validación como conocimiento que amerita su transmisión generacional, sino de que sea llevado a las aulas universitarias, de que se actualice. Sin embargo, estos conocimientos van significando el desempeño y generan nuevas exigencias que no son satisfechas por la cultura científico investigativa que lo originó, y hacen que el desempeño investigativo no sea sostenible; aquí se da el momento crítico de la contradicción. Una vez asimilados estos nuevos saberes, se enriquece la cultura científico investigativa, se pone a tono con las exigencias de desempeño y se supera la contradicción, que pasa a un nuevo ciclo de desarrollo.

Esta cultura científico investigativa es portadora de los elementos esenciales inherentes a los contenidos de la profesión, a los modos de actuar, a la regulación de la conducta. Todo lo relacionado con la investigación, a los componentes fundamentales de los distintos procesos básicos, en general, porta toda una riqueza cultural de la que debe apropiarse el sujeto interiorizándola y expresándola posteriormente en un desempeño investigativo idóneo, que es el causante de la generación de cambios como los ya mencionados de tipo tecnológicos, gnoseológicos, etc., los que modifican esa cultura interiorizada por el individuo, actualizándola y enriqueciéndola y esto trasciende a lo ya establecido, negándolo.

No obstante, ambos se dan en unidad, en tanto la diversidad y unicidad de la cultura científico investigativa se preserva mediante la interiorización, en el desarrollo que ha provocado en ella los cambios resultantes del desempeño investigativo idóneo como contrario dialéctico de la interiorización de la diversidad y unicidad de la cultura científico investigativa.

Las relaciones que se establecen en este proceso de formación investigativa basada en competencias entre el estudiante y el profesor, el estudiante y otros estudiantes, el estudiante y los profesionales en ejercicio, el estudiante y otros elementos del entorno socio afectivo y en general del entorno social de desarrollo, al efectuar las actividades investigativas y las de índole académica y laboral, en la actividad y la comunicación, son interiorizadas y matizadas a través de interpretaciones, 
configuraciones y rasgos personales, convirtiéndolas en propias del sujeto, logrando la interiorización de la cultura científico investigativa, lo que al mismo tiempo imprime un sello personal al desempeño.

1.2.3. Estrategia para la implementación del modelo para la formación investigativa basada en competencias La estrategia propuesta toma elementos de la planeación estratégica y dirección por objetivos, pero se desarrolla desde el currículo en el proceso de enseñanza aprendizaje, lo que le confiere un carácter didáctico.

El objetivo general de la estrategia consiste en favorecer el desempeño investigativo de los ingenieros industriales mediante la transformación cualitativa del proceso de formación investigativa, sustentado en la formación de competencias investigativas.

La estrategia consta de seis fases;

\section{FASE I: Diagnóstico del potencial para la formación investigativa y determinación de las competencias que} sustentan este proceso

Momento 1. Diagnóstico del potencial para la formación investigativa basada en competencias

Momento 2. Determinación de las competencias investigativas

En este se procede a determinar las competencias investigativas que sustentan la formación investigativa competente del ingeniero industrial, las que si somos consecuentes con la clasificación asumida, son consideradas competencias profesionales básicas dado su grado de generalidad y/o expresión en el desempeño de disímiles profesiones, pero que adquieren una significación especial a partir de su aplicación a los diversos procesos básicos de la Ingeniería Industrial.

FASE II: Identificación y determinación de escenarios profesionales, niveles de formación investigativa y direcciones estratégicas de formación

Momento 1. Identificación de escenarios profesionales

Momento 2. Identificar los niveles de formación investigativa competente

Momento 3. Determinar las direcciones estratégicas formativas y los grupos implicados

\section{FASE III: Planificación y organización de la formación investigativa basada en competencias}

Esta fase tiene como propósito fundamental el aseguramiento de las condiciones para aquellas disciplinas y asignaturas que tributan a la configuración de las competencias investigativas.

\section{FASE IV: Ejecución de la formación investigativa competente}

Momento 1. Dirección estratégica orientada a la preparación teórica, metodológica y tecnológica del personal docente Las acciones en esta dirección tienen como objetivo la preparación del personal docente en la formación basada en competencias profesionales en los contextos universitarios, para dirigir la formación investigativa de los estudiantes de modo que logren la formación y desarrollo de las competencias investigativas, que favorezcan un desempeño investigativo idóneo.

Momento 2. Dirección estratégica orientada a la formación investigativa basada en competencias

Las acciones en esta dirección tienen como objetivo propiciar la configuración de las competencias investigativas que sustentan la formación investigativa competente del ingeniero industrial, las que ven favorecida su formación por el empleo de manera combinada en el proceso de enseñanza aprendizaje del estudio de casos de situaciones profesionales y los proyectos de Ingeniería Industrial. 


\section{FASE V: Evaluación y certificación del nivel de formación investigativa}

Momento 1. Evaluación: Evaluar competencias se constituye en un proceso complejo y dinámico que permite constatar las evidencias de estas, demostradas en condiciones reales o simuladas, lo más cercano posible a los contextos profesionales, de conformidad con el desempeño descrito en relación con las competencias investigativas. La misma presupone la emisión de juicios de valor sobre los resultados logrados, teniendo en cuenta los procesos puestos en práctica para obtenerlos y la relación costo beneficio de la actividad, los que se recogen en un dictamen final emitido por el comité evaluador creado al efecto, en el que se indica el nivel de formación alcanzado por el evaluado.

Momento 2. Certificación: El propósito fundamental del proceso de certificación es otorgar el reconocimiento al estudiante de las competencias que se han configurado en él o del nivel logrado de formación; lo cual es, sin dudas, una síntesis en el proceso de formación referido a un período determinado del mismo; sin embargo no constituye en modo alguno un punto final, porque dado su carácter sistemático y relacional con los demás procesos, adquiere la cualidad de una validación continua durante la vida del sujeto. La certificación lleva en su esencia la evaluación de las competencias. En este sentido se toman los momentos establecidos por R. Tejeda y P. Sánchez (2008) que se corresponden con el proceso de formación inicial:

\section{FASE VI: Retroalimentación del proceso de formación investigativa basado en competencias}

Esta fase es concebida como esencial para la mejora continua del proceso de formación investigativa competente y para garantizar el impacto del mismo en el estudiante durante el desarrollo de las competencias investigativas.

La estrategia prevé en dependencia de la sostenibilidad o no del proceso formativo, mantener las direcciones formativas estratégicas y el rediagnóstico inicial del potencial con vistas a modificar esencialmente estas direcciones en consonancia con los requerimientos socioprofesionales.

\section{Metodología}

En el estudio realizado se seleccionó una muestra de 26 estudiantes coincidente con la población. Entre los principales métodos utilizados sobresalen el método sistémico y la modelación. La experimentación sobre el terreno se empleó para lograr la aplicación parcial de la estrategia para la formación investigativa basada en competencias.

Dentro de las técnicas estadísticas: se utilizó el cálculo de frecuencia absoluta y relativa, el cálculo del coeficiente de competencia de los expertos ( $k$ ) y el método Delphi que permitió determinar el nivel de concordancia de los expertos que fueron sometidos a consultas con relación a la factibilidad y pertinencia del modelo y de la estrategia propuesta.

Se empleó la prueba de libre distribución Ji- cuadrado con la finalidad de contrastar las frecuencias observadas (Fo) con las frecuencias esperadas(Fe) de acuerdo con la hipótesis planteada.

\section{Resultados}

Concluida la puesta en práctica de la estrategia formativa según lo previsto, se procedió a valorar la posibilidad de aplicación exitosa de la misma en la práctica educativa, o sea, su factibilidad. Para ello fueron tenidos en cuenta los criterios siguientes:

- La utilidad que tiene para los gestores del proceso la estrategia para la formación investigativa del ingeniero industrial basada en competencias.

- Actualidad y pertinencia de la propuesta. 
- Aspectos que han mejorado en el proceso de formación investigativa al aplicar la estrategia.

Estos criterios fueron avalados por el grado de satisfacción mostrado por los gestores del proceso al responder el cuestionario para recopilar información luego de la aplicación parcial de la estrategia para la formación investigativa basada en competencias, dado que todos los aspectos fueron evaluados con un elevado porcentaje de significación, en cuanto a muy útil y útil. A continuación se exponen los principales resultados:

- Las etapas de la estrategia son consideradas muy útiles o útiles por el $95 \%$ de los encuestados.

- El 100\% de los encuestados considera que la propuesta tiene un alto grado de pertinencia y actualidad.

- Los criterios determinados para el diagnóstico del potencial para la formación investigativa del ingeniero industrial basada en competencias, el $95 \%$ evalúa entre muy útiles o útiles.

- El empleo de situaciones profesionales reales para desarrollar los estudios de casos fue considerado por el $100 \%$ de los encuestados como muy útil.

- El empleo de situaciones profesionales portadoras de disyuntivas axiológicas para favorecer la formación de valores relacionados con la formación investigativa fue valorado como muy útil por el $83 \%$ de los encuestados y como útil por el $17 \%$ restante.

Los encuestados consideran que los aspectos que mejoraron como resultado de la aplicación de la estrategia fueron:

- Motivación hacia la investigación, entendiendo esta como una forma de lograr una mejor formación profesional.

- Empleo de TIC, para realizar la gestión de información relacionada con los proyectos y con los casos de situaciones profesionales sometidas a estudio.

- Conductas y actitudes adecuadas asociadas a la investigación, asumidas por los estudiantes durante el desarrollo de las actividades relacionadas con esta.

- Elevación del nivel en la identificación y presentación de los problemas profesionales detectados, así como en la argumentación de los mismos y de las causas que los originan.

- Participación activa de los estudiantes en la búsqueda de elementos teórico conceptuales que le permitan definir y analizar, desde la diversidad e integración disciplinar de la carrera, el problema profesional, analizar y seleccionar las alternativas de solución, diseñar la solución e implementarla.

- Se considera que el nivel de integración entre lo académico, laboral e investigativo en el proceso de formación del ingeniero industrial mejoró, de acuerdo con los criterios del $90 \%$ de los encuestados.

En la pregunta relacionada con la fase de la estrategia que resultó más difícil aplicar, el $57 \%$ de los encuestados señaló la fase cinco y el $43 \%$ la seis, evaluación y retroalimentación, respectivamente, la evaluación por el nivel de preparación que requiere, y la retroalimentación por el grupo de categorías involucradas, lo cual es representativo de la complejidad del proceso. A pesar de esto, la observación sistemática al desarrollo de las fases y acciones permitió afirmar que la estrategia es factible de aplicarse y su aplicación en todo el ciclo de formación aportaría mayor cantidad de elementos que enriquecerían tanto la evaluación como la retroalimentación, lo que fue demostrado si se tienen en cuenta los aspectos siguientes:

- Se favoreció la formación de las competencias investigativas en correspondencia con el nivel de configuración inicial.

- Se favoreció la interacción y la coherencia de las acciones realizadas por los diferentes gestores del proceso.

- Los profesores elevaron su preparación para llevar a cabo el proceso, lo que permite afirmar la factibilidad en sus etapas y acciones.

- Existió un alto grado de cumplimiento de los objetivos, fases y acciones generales previstas.

- Posibilitó la integración de los componentes académicos, laboral e investigativo, con mayor preparación de todos los implicados.

Las insuficiencias presentes durante la aplicación de la estrategia formativa están relacionadas fundamentalmente con la resistencia al cambio, al transitar de un proceso de formación investigativa dirigido a desarrollar habilidades a uno de mayor complejidad dirigido a la formación de competencias investigativas. 


\subsection{Comparación entre grupos}

Para culminar la experimentación se realizó un estudio comparativo entre dos grupos de estudiantes, conformados lo más homogeneamente posible, sobre todo en lo que respecta a su rendimiento académico, actitud. y aptitud ante el estudio entre otros aspectos, y tomando como elemento esencial de comparación, el grado de formación investigativa alcanzado al concluir el nivel de configuración inicial del sistema de competencias investigativas, el que como ya se ha mencionado anteriormente comprende los dos primeros años de la carrera.

Los resultados del primer grupo, denominado Grupo 1, se enmarcan en cursos previos a la aplicación parcial de la estrategia, primer año. El otro es denominado como Grupo 2 y sus resultados corresponden al período posterior a la aplicación parcial de la estrategia.

Para ello se aplicó la prueba de hipótesis Ji-cuadrado con la finalidad de contrastar las frecuencias observadas (Fo) con las frecuencias esperadas(Fe), a tal efecto se plantean las hipótesis estadísticas $H_{\circ}$ y $H_{1}$ : $\mathrm{H}_{0}$ : Las frecuencias observadas son iguales a las esperadas. ( $\mathrm{Fo}=\mathrm{Fe}$ ).

$\mathrm{H}_{1}$ : Las frecuencias observadas difieren de las esperadas. (Fo $\neq \mathrm{Fe}$ ).

Traduciendo estas hipótesis al lenguaje pedagógico en el contexto del problema, podemos plantearlas como: $\mathrm{H}_{0}$ : El nivel de formación investigativa evidenciado por el Grupo 1 previo a la aplicación de la estrategia, es el mismo al evidenciado para el Grupo 2, con posterioridad a la aplicación de la estrategia.

$\mathrm{H}_{1}$ : El nivel de formación investigativa evidenciado por el Grupo 1, previo a la aplicación de la estrategia, no es el mismo al evidenciado para el Grupo 2 con posterioridad a la aplicación de la estrategia. Los resultados obtenidos para un nivel de significación de $\alpha=0.05$ se muestran a continuación:

Tabla 1

Frecuencias observadas

\begin{tabular}{|c|c|c|c|c|}
\hline \multicolumn{5}{|c|}{$\begin{array}{c}\text { Frecuencias } \\
\text { observadas }\end{array}$} \\
\hline Nivel de configuración & Alto & Medio & Bajo & Total \\
\hline Grupo 1 & 8 & 9 & 9 & 26 \\
\hline Grupo 2 & 14 & 10 & 2 & 26 \\
\hline Total & 22 & 19 & 11 & 52 \\
\hline
\end{tabular}

Tabla 2

Frecuencias esperadas estimadas. $\chi^{2}$

\begin{tabular}{|c|c|c|c|c|}
\hline & \multicolumn{5}{|c|}{ Frecuencias } & esperadas & estimadas \\
\hline Nivel de configuración & Alto & Medio & Bajo & Total \\
\hline Grupo 1 & 11 & 9.5 & 5.5 & 26 \\
\hline Grupo 2 & 11 & 9.5 & 5.5 & 26 \\
\hline Total & 22 & 19 & 11 & 52 \\
\hline
\end{tabular}


Tabla 3

Cálculo del estadístico $\chi^{2}$

\begin{tabular}{|c|c|c|c|c|c|c|}
\hline \multicolumn{7}{|c|}{ Estadístico 72} \\
\hline Oi-Ei & -3 & 3 & -0.5 & 0.5 & 3.5 & -3.5 \\
\hline$(\mathrm{Oi}-\mathrm{Ei}) 2$ & 9 & 9 & 0.25 & 0.25 & 12.25 & 12.25 \\
\hline$(\mathrm{Oi}-\mathrm{Ei}) 2 / \mathrm{Ei}$ & 0.818181818 & 0.818181818 & 0.026315789 & 0.026315789 & 2.227272727 & 2.227272727 \\
\hline
\end{tabular}

Valor calculado $\chi^{2}=6,143540$

Valor teórico $\chi^{2} 0,95(2)=5.591$

$\chi^{2}=6,143>\chi^{2} 0,95(2)=5,591$

En vista de que el valor calculado $\chi^{2}$ es mayor que el valor teórico $\chi^{2} 0,95$ (2), por tanto se rechaza la hipótesis nula $\left(\mathrm{H}_{0}\right.$ : $\left.\mathrm{Fo}=\mathrm{Fe}\right)$ con una $\mathrm{p}<0,05$.

Lo anterior permite concluir en términos estadísticos que, existen diferencias en la frecuencia del nivel de formación investigativa (Alto - Medio - Bajo) evidenciado por los estudiantes del Grupo 1 previo a la aplicación de la estrategia y la frecuencia del nivel de formación investigativa (Alto - Medio - Bajo) evidenciado por los estudiantes del Grupo 2 con posterioridad a la aplicación de la estrategia. Es de destacar que los resultados observados en el nivel de formación investigativa alto, son superiores en el Grupo 2 (14) con respecto al Grupo 1 (8), al mismo tiempo que los bajos son significativamente inferiores, para el Grupo 2 (2) con respecto al Grupo 1 (9), y se observa mayor equilibrio en los valores medio para ambos grupos, los que son 9 y 10 respectivamente, con una diferencia de sólo uno, lo que permite afirmar que los resultados obtenidos en el Grupo 2 son superiores a los alcanzados en el Grupo 1, en lo que respecta a la formación investigativa.

Esto se traduce en que las evidencias proporcionadas por los grupos comparados son suficientes para afirmar que el nivel de formación investigativa mostrado por el Grupo 1, previo a la aplicación de la estrategia, no es el mismo que el mostrado por el Grupo 2 con posterioridad a la aplicación de la estrategia. Estos resultados constituyen argumentos a favor de la verificación de la hipótesis de investigación planteada inicialmente:

$\mathrm{H}_{\mathrm{i}}$ : Una estrategia sustentada en un modelo para la formación investigativa basada en competencias profesionales que tome en consideración la relación entre la interiorización de la cultura científico investigativa de la ingeniería industrial y el desempeño investigativo, mediada por el estudio de casos y los proyectos de ingeniería, deberá favorecer el desempeño de los estudiantes en la solución de problemas inherentes a los procesos básicos de la profesión.

\section{Conclusiones}

Los resultados obtenidos al ejecutar las tareas de investigación permiten formular las conclusiones siguientes:

En el contexto de las transformaciones que tienen lugar en la universidad, la formación investigativa que caracteriza al profesional de la carrera de Ingeniería Industrial, sustentada en y desde los procesos básicos de la misma (Gestión de la Calidad, Logística Empresarial, Gestión de Procesos y Gestión de Recursos Humanos), viene a satisfacer las exigencias de una formación integral que garantice el modo de actuación de este, en concordancia con las exigencias relativas a su desempeño profesional y en particular investigativo.

Las valoraciones efectuadas y los resultados obtenidos con la aplicación del método de experimentación sobre el terreno en la carrera de Ingeniería Industrial, permiten concluir que la estrategia, la cual materializa en la práctica el modelo para el proceso de formación investigativa basada en competencias, puede ser aplicada en todo el ciclo de formación inicial y posibilita planificar, organizar, ejecutar y evaluar de manera integral y coherente este proceso, garantizando su sostenibilidad formativa y la formación de las competencias investigativas del ingeniero industrial. 


\section{Referencias bibliográficas}

Barrera Kalhil, J. (2003). Estrategia pedagógica para el desarrollo de habilidades investigativas en los estudiantes de Ciencias Técnicas. Tesis en opción al grado científico de Doctor en Ciencias Pedagógicas. Centro Universitario José Antonio Echeverría. La Habana, p.1.

Castro Sánchez, F. (1999). "El estudio de los problemas sociales de la Ciencia y la Tecnología y la formación del profesional ingeniero". Revista Educación Universitaria, no. 2. Matanzas, Cuba, pp.143-144.

Cruz Baranda, S. y Fuentes González, H. (2003). "La didáctica de la investigación científica." CeeS Manuel F. Gran. Universidad de Oriente. Santiago de Cuba, p.3.

Fuentes, H., E. Matos y J. Montoya (2007). El proceso de investigación científica orientada a la investigación en ciencias sociales. Guaranda, Provincia de Bolívar, Venezuela, p.117.

Fuentes, H.(2004). La Sociedad del conocimiento: Nuevos retos a la Universidad. CeeS Manuel F. Gran, Universidad de Oriente, Santiago de Cuba, p.6.

Horruitiner Silva, P. (2006). La Universidad Cubana: el modelo de formación. Editorial Félix Varela, Ciudad de la Habana, p.27.

Moreno González, N. M. y F. O. Rodríguez González (2002). "La gestión de la información como base de la gestión del conocimiento." Revista Cubana de Educación Superior, vol. XXII, no 2, p.20.

Núñez Jover, J. (1999). La Ciencia y la Tecnología como procesos sociales, Editorial "Félix Varela". La Habana. , p.89.

Samaniego Villareal, N. (1996). “Competencias Laborales y Mercado de trabajo. Los desafíos de una nueva era posterior a la revolución industrial”. México, p.4.

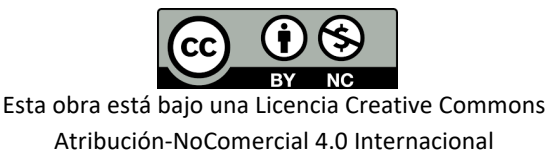

